University of Nebraska - Lincoln

DigitalCommons@University of Nebraska-Lincoln

Ralph Skomski Publications

Research Papers in Physics and Astronomy

March 2008

\title{
Temperature dependence of half-metallic hybridization gaps
}

\author{
Ralph Skomski \\ University of Nebraska-Lincoln, rskomski2@unl.edu
}

Follow this and additional works at: https://digitalcommons.unl.edu/physicsskomski

Part of the Physics Commons

Skomski, Ralph, "Temperature dependence of half-metallic hybridization gaps" (2008). Ralph Skomski Publications. 54.

https://digitalcommons.unl.edu/physicsskomski/54

This Article is brought to you for free and open access by the Research Papers in Physics and Astronomy at DigitalCommons@University of Nebraska - Lincoln. It has been accepted for inclusion in Ralph Skomski Publications by an authorized administrator of DigitalCommons@University of Nebraska - Lincoln. 


\title{
Temperature dependence of half-metallic hybridization gaps
}

\author{
Ralph Skomski ${ }^{\text {a) }}$ \\ Department of Physics and Astronomy and Nebraska Center for Materials and Nanoscience, \\ University of Nebraska, Lincoln, Nebraska 68588, USA
}

(Presented on 6 November 2007; received 13 September 2007; accepted 4 November 2007; published online 10 March 2008)

\begin{abstract}
The temperature dependence of the spin polarization of half-metallic ferromagnets is investigated by model calculations. Emphasis is on changes in the density of states, especially on the closure of the hybridization gap. The Stoner-type calculation yields a simple one-electron expression for the gap as a function of temperature and a corresponding transition temperature $T^{*}$, above which the spin polarization is drastically reduced. The small parameter determining the finite-temperature breakdown of the spin splitting is the width of the hybridization gap relative to other bandwidth parameters. The model predictions are consistent with the experiment and with first-principles electronic structure calculations, and we argue that such band-structure effects are not limited to specific materials but rather widespread in hybridization-gap half-metals. (C) 2008 American Institute of Physics. [DOI: 10.1063/1.2836678]
\end{abstract}

\section{INTRODUCTION}

Half-metallic ferromagnets have attracted much attention as potential materials for spin electronics because they only spin channel for conduction. ${ }^{1,2}$ However, the pronounced temperature dependence of the spin polarization has been a long-standing question. Since spin-electronic devices and sensors are typically used at room temperature, this is a practically important issue. While spin mixing causes the opening of a second conduction channel at arbitrarily low temperatures, ${ }^{3}$ the reduction of the spin polarization is more pronounced than expected from the spontaneous magnetization of simple ferromagnets. It may be described by a transition temperature $T^{*}<T_{C}$, above which the half-metallic spin polarization is strongly reduced. This behavior is partially explained by the noncollinear spin structure of many half-metallics linked to the presence of two or more crystallographically nonequivalent sites and to phononic excitations, ${ }^{4}$ but the question arises as to whether there is a more general reason for the strongly reduced finitetemperature spin polarization. Figure 1 shows schematic $\uparrow$ and $\downarrow$ densities of states (DOS) for a typical half-metal at zero temperature, characterized by the absence of $\downarrow$ states at the Fermi level. At nonzero temperatures, the Fermi surface seems to contain not only $\uparrow$ but also some $\downarrow$ electrons.

One possible explanation is nonquasiparticle states due to electron correlations. ${ }^{5}$ However, spin-dependent electronic-structure calculations on $\mathrm{CrO}_{2}$ (Ref. 6) and $\mathrm{NiMnSb}$ (Ref. 7) indicate that the independent-electron approximation works reasonably well for typical half-metallics, and it has been emphasized that the corresponding corrections are particularly large for broad transition-metal bands, where correlations are least important. ${ }^{8}$

A characteristic challenge is that half-metals a rather inhomogeneous class of magnetic materials, both structurally and electronically. Our focus is on hybridization-gap half-

${ }^{a)}$ Electronic mail: rskomski@neb.rr.com metals such as $\mathrm{CrO}_{2}$ and $\mathrm{NiMnSb}$, which form themselves several subclasses. ${ }^{2,9}$ In a nutshell, are there common finitetemperature features shared, for example, by half-metallic oxides and semi-Heusler alloys? Why does it seem that nature "abhors half-metallicity." 10

Spin mixing is a rather common feature but only partially explains the temperature dependence of the spin polarization. In fact, spin mixing means that the $\uparrow$ DOS is "mirrored" in the $\downarrow$ DOS, without explicit consideration of changes in the DOS. ${ }^{3}$ Both numerical simulations ${ }^{7}$ and model calculations ${ }^{8}$ indicate substantial deformations of the DOS. While a full analysis of these changes goes far beyond the scope of this work, the present paper focuses on one important aspect of the problem, namely, on the closure of the hybridization gap at finite temperatures (Fig. 2).

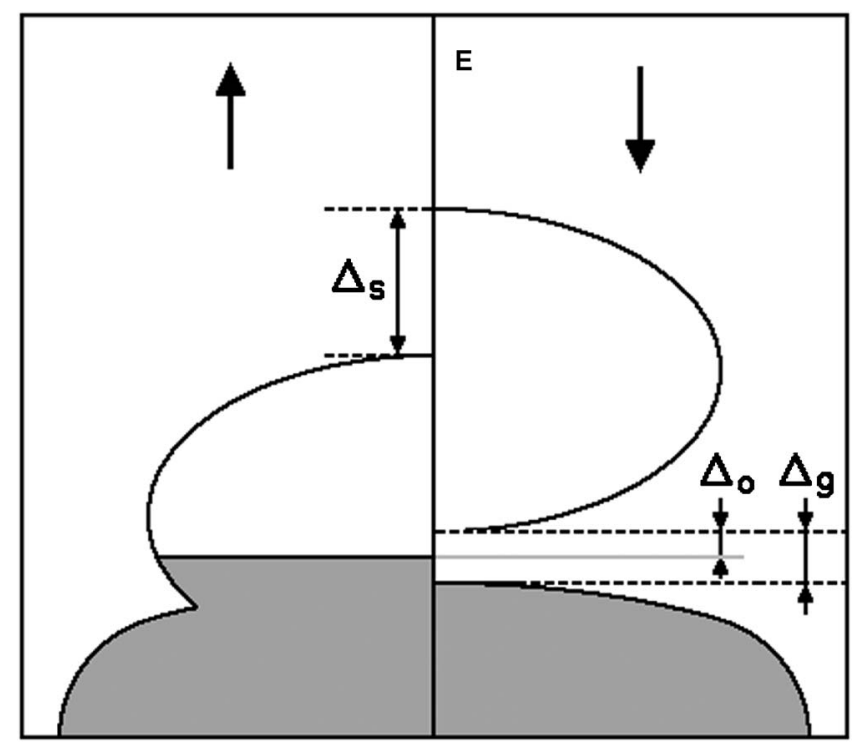

FIG. 1. Schematic densities of state $D_{\sigma}(E)$ for the two spin directions $\uparrow$ and $\downarrow$. 


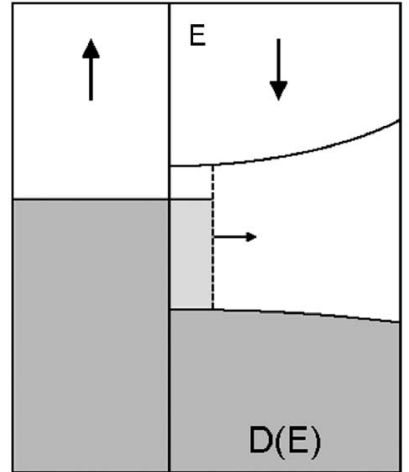

(a)

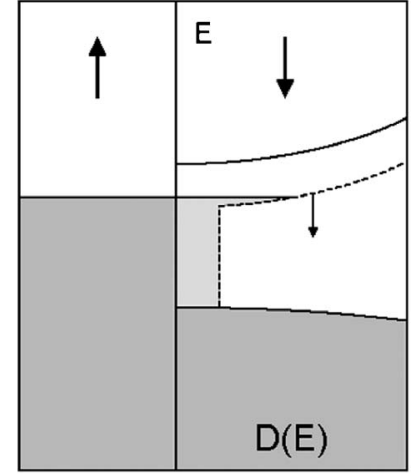

(b)
FIG. 2. Finite-temperature modification of DOS: (a) spin mixing and (b) gap closure.

\section{MODEL AND RESULTS}

The present analysis is based on the well-known Stoner model of ferromagnetism, which ascribes ferromagnetism to an effective interaction field reflecting the Coulomb interaction $U \sim I$ of the electrons. In terms of particle number operators, $E_{C}=U n_{\uparrow} n_{\downarrow}$ or, with $m=n_{\uparrow}-n_{\downarrow}$ and $n=n_{\uparrow}+n_{\downarrow}$,

$$
E_{C}=\frac{U}{4}\left(n^{2}-m^{2}\right) \text {. }
$$

This equation shows how the Coulomb interaction favors ferromagnetism. In the quantum-mechanical mean-field approximation, one assumes that $m^{2} \sim m\langle m\rangle$ and obtains a splitting $\pm E_{0}$ between $\uparrow$ and $\downarrow$ bands (Fig. 2). In this approximation, which is used in both model calculation and numerical electronic-structure simulations, the exchange splitting is proportional to $m U$ or, in the Stoner model, $2 E_{0}$ $=M I$, where $I \sim U$ is the Stoner parameter (about $1 \mathrm{eV}$ ) and $M$ is the magnetization measured in $\mu_{B}$ /atom. Note that $I$ is usually referred to as a the Stoner exchange integral, although the Coulomb interaction [Eq. (1)] is often the leading contribution.

It is important to keep in mind that Eq. (1) refers to a single atom, and neither $\langle m\rangle$ nor $\langle m\rangle$ are necessarily homogeneous in solids. Essentially, $\langle m\rangle$ is a local quantummechanical average that may vary from atom, for example, in antiferromagnets and at finite temperatures. ${ }^{8}$ The Stonertype assumption of a homogeneous magnetization ignores spin fluctuations and substantially overestimates the Curie temperature. ${ }^{11}$ However, here, we are not interested in spin fluctuations, which give rise to the above-mentioned spin mixing, but in the opposite limit. From Fig. 3, we see that the gap obeys

$$
\Delta=2 E_{0}+\Delta_{r}-W / 2 .
$$

In the present model, $E_{0}=M I / 2$ is temperature dependent because $M$ decreases as $T_{C}$ is approached. At zero and nonzero temperatures, Eq. (2) becomes $\Delta_{0}=M_{0} I+\Delta_{r}-W / 2$ and $\Delta(T)=M(T) I+\Delta_{r}-W / 2$, so that

$$
\Delta(T)=\Delta_{0}+I\left[M(T)-M_{0}\right] .
$$

Gap closure and the vanishing of half-metallic ferromagnetism occur for $\Delta\left(T^{*}\right)=0$. Since the gap is usually smaller

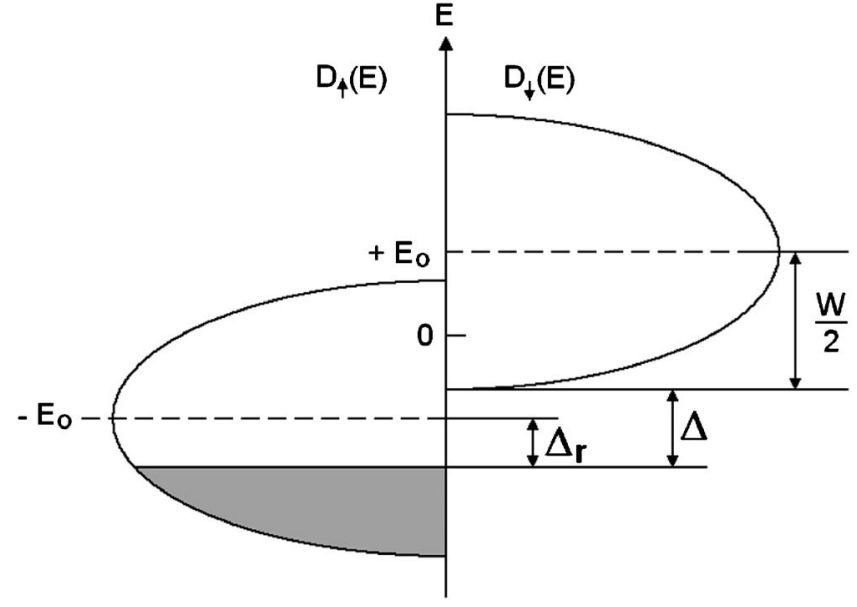

FIG. 3. Gap $\Delta$ and its relation to the exchange splitting $E_{0}$.

than $I M_{0}$, this happens well below $T_{C}$, even for gaps where $\Delta_{0} / k_{B}$ is much larger than $T_{C}$. Figure 4 shows the transition temperature $T^{*}$ as a function of the gap width. Note that $T^{*}$ strongly depends on the details of $M(T)$. As one can see from the inset, $T^{*}$ in Fig. 4 corresponds to a simple mean-fieldtype $M(T)$ dependence with $T_{C}=700 \mathrm{~K}$. However, halfmetallic ferromagnets have two or more crystallographically nonequivalent sublattices, which lead to a reduced magnetization at intermediate temperatures. ${ }^{12}$ Inserting the corresponding "inflected" $M(T)$ curves yields a reduction of $T^{*}$ for intermediate gap widths.

A slightly different approach is to consider the halfmetals as weak ferromagnets, where thermal excitations lead to the population of minority $(\downarrow)$ states. $^{8}$ The number of $\uparrow$ and $\downarrow$ electrons per atoms obeys $N_{\uparrow, \downarrow}=\int D\left(E \pm E_{0}\right) \mathrm{d} E$, where the integral extends from $-\infty$ to $E_{F}$. For rectangular bands, $M=N_{\uparrow}-N_{\downarrow}=2 E_{0} D$ or $E_{0}=M / 2 D$. The temperature dependence of $M$ leads to a temperature dependence of $E_{0}$, corresponding to a gap closure when $\Delta_{0} \sim E_{0}(0)-E_{0}(T)$. Since $D \sim 1 / W$, the small parameter of this approach is $\Delta_{0} / W$.

\section{DISCUSSION AND CONCLUSIONS}

Section II shows that hybridization gaps tend to close far below the Curie temperature. From Fig. 4 we estimate that

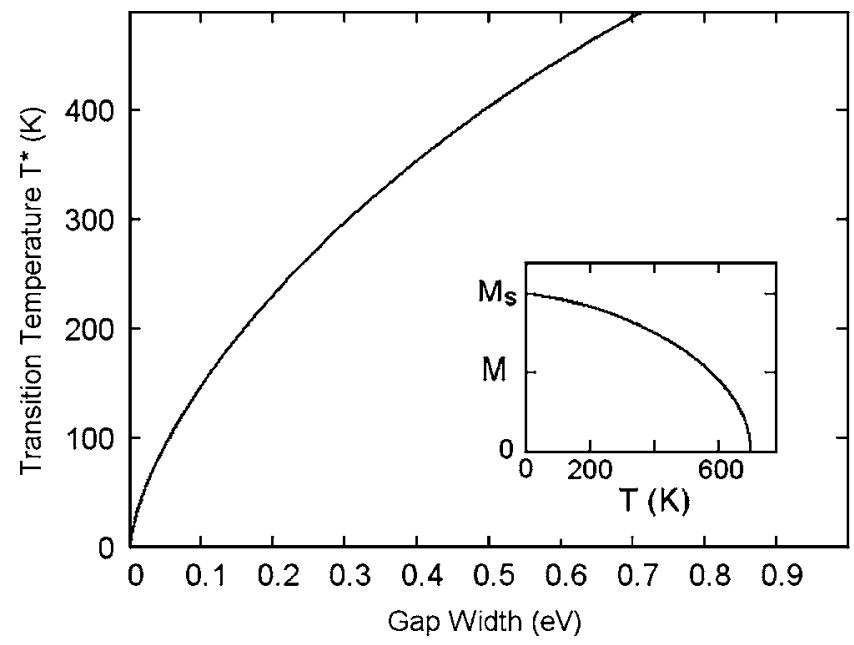

FIG. 4. Transition temperature $T^{*}$ at which the half-metallic ferromagnetism breaks down. The inset shows the assumed $M(T)$ curve. 
$T^{*}$ is typically around or below room temperature, in agreement with experiment. The involved small parameter is the zero-temperature hybridization gap, as compared to other bandwidth parameters, such as the exchange splitting and the $3 d$ bandwidth. Typical gaps are much bigger than $k_{B} T$ but smaller than the mentioned band-structure energy parameters, so that relative small finite-temperature changes in the DOS translate into disproportionally strong gap changes.

The gap reduction akin to band-structure effects considered in Ref. 7 adds to the spin-mixing effect. To a large extent, this is a basic result and is independent of the details of the calculations. For example, the coherent potential approximation used in Ref. 7 is known to poorly describe band edges because it is a single-site theory and ignores cluster localization. ${ }^{13}$ In the present context, cluster localization means that there are regions with nearly parallel atomic moments and that itinerant electrons gain extra kinetic energy by hopping inside these finite-size regions or "clusters." A similar effect is created by noncollinear magnetization states, ${ }^{4,12}$ where the electrons experience a spin-dependent potential that varies over a few interatomic distances. However, spin-mixing and noncollinear effects add to the bandstructure effects considered here and do not invalidate the gap-closure mechanism of Sec. II.

In fact, Sec. II represents an even simpler approximation, namely, the virtual crystal approximation (VCA). In this approximation, known from alloys ${ }^{13}$ the true potential $V(\mathbf{r})$ is replaced by an average $\langle V\rangle$. In the original alloy analogy, $\langle V\rangle=p_{A} V_{A}+p_{B} V_{B}$, where $A$ and $B$ denote different elements, ${ }^{13}$ where at finite temperatures, the averaging refers to thermally randomized spin-dependent potentials. ${ }^{14}$ Quantitatively, the VCA is a very crude and somewhat oversimplified approach, but it becomes exact in the presently considered limit of strong interatomic hopping. Physically, the hopping ensures that the $3 d$ electrons experience an average potential $\langle V\rangle$ rather than temporarily residing on individual atoms. This must be contrasted to tight-binding spin mixing, ${ }^{3}$ where the hopping is considered as a small perturbation.

In summary, the present semiquantitative calculations provide a physical explanation of the temperature $T^{*}$ and are consistent with recent band-structure calculations ${ }^{7}$ and with the experiment. ${ }^{2}$ The gap-closure mechanism investigated in this paper is rather general and is expected to apply to a broad variety of half-metallic ferromagnets, although nu- merical electronic-structure calculations will be needed to reach a quantitative structure-specific understanding of the phenomenon. While the present approach is only semiquantitative, it captures an essential feature of a wide range halfmetallics, namely, that hybridization gaps are rather narrow and strongly affected by seemingly small thermally or otherwise induced changes in the DOS.

In summary, we have investigated how the closure of the hybridization gap affects the finite-temperature spin polarization of half-metallic ferromagnets. We have obtained a simple analytical expression for the temperature dependence of the hybridization gap and found that the gap closes at transition temperatures $T^{*}$ generally much lower than $T_{C}$. The gap reduction is a one-electron rather than a correlation effect and adds to the spin mixing. The present theory cannot replace detailed structure-specific numerical calculations, but it yields a rather general explanation of the strongly reduced finite-temperature magnetization of half-metallic ferromagnets.

\section{ACKNOWLEDGMENTS}

This work has been supported by NSF-MRSEC and NCMN. Thanks are due to P. A. Dowben for discussing various aspects of half-metallic ferromagnetism.

${ }^{1}$ R. A. de Groot and K. H. J. Buschow, J. Magn. Magn. Mater. 54-57, 1377 (1986).

${ }^{2}$ J. M. D. Coey and M. Venkatesan, J. Appl. Phys. 91, 8345 (2002).

${ }^{3}$ R. Skomski and P. A. Dowben, Europhys. Lett. 58, 544 (2002).

${ }^{4}$ P. A. Dowben and R. Skomski, J. Appl. Phys. 95, 7453 (2004).

${ }^{5}$ V. Yu. Irkhin, M. I. Katsnelson, and A. I. Liechtenstein, J. Phys.: Condens. Matter 19, 315201 (2007).

${ }^{6}$ I. I. Mazin, D. J. Singh, and C. Ambrosch-Draxl, Phys. Rev. B 59, 311 (1999).

${ }^{7}$ M. Ležaić, Ph. Mavropoulos, J. Enkovaara, G. Bihlmayer, and S. Blügel, Phys. Rev. Lett. 97, 026404 (2006).

${ }^{8}$ R. Skomski, J. Phys.: Condens. Matter 19, 315202 (2007).

${ }^{9}$ C. M. Fang, G. A. de Wijs, and R. A. de Groot, J. Appl. Phys. 91, 8340 (2002).

${ }^{10}$ K. Hathaway, as quoted in P. A. Dowben, J. Phys.: Condens. Matter 19, 310301 (2007).

${ }^{11}$ T. Moriya, Spin Fluctuations in Itinerant Electron Magnetism (Springer, Berlin, 1985)

${ }^{12}$ R. Skomski, J. Zhou, P. A. Dowben, and D. J. Sellmyer, J. Appl. Phys. 97, 10C305 (2005)

${ }^{13}$ E. N. Economou, Green's Functions in Quantum Physics (Springer, Berlin, 1979).

${ }^{14}$ V. A. Gubanov, A. I. Liechtenstein, and A. V. Postnikov, Magnetism and the Electronic Structure of Crystals (Springer, Berlin, 1992). 